\title{
Poésie
}

\section{Paul Hassoun}

\section{De pierres et de chairs}

L'Ã@t $\mathrm{A} \bigodot$ qui demeure ne prend de moi qu'un sang de passage

des nuages se figent

$\tilde{A} @$ tales dans la marÃ $(e$ des vents

Stables soudain, des temps s'amoncellent

le flux des immẤ(C) diatetÃ@)s surprend mon regard

des secondes en gangues s'Ã $@$ garent

- diamants d'instants enchevÃatrÃ@s -

dans l'irrÃ $(a)$ alit $\tilde{A} \Subset$ de tes yeux,

nos vies fracturÃ@es glissent et divaguent

Alors

alors, imprÃ@) visible tu surgis ma mort au go $\tilde{A} » t$ de mangue verte un matin $d \tilde{A} @$ $@$ vorÃ@e

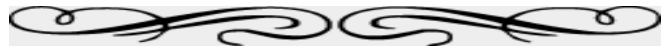

\section{Herbes et rocs}

Sur la toile rÃache o $\tilde{A}^{1}$ l'improbable jaillit

- mesures d'un temps dÃ@jÃ trop dÃ@comptÃ@ -

nos rÃaves s'espacent qui demeurent enfouis

(et vogue le navire

d'oÃ1 la pi $\tilde{A}^{*}$ ce fut jet $\tilde{A}(e)$

Rouges, des marches entrouvrent l'espace marin

Ã@chelons verticaux descendant vers le vide

nul nÃ@ jamais ne les gravit

mais qui donc ne les descend?

(somptueuse, voluptueuse folie nourrie de nos $d \tilde{A} @$ Clires

aux aguets tu te tiens)

Construites de nos mains tendues

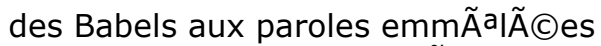
attendent que la mer les dÃ $\odot$ vore

Blotti dans le silence de runes en vain implorÃ@es 
j'apprã $@$ hende mes nuits

o $\tilde{A}^{1}$, aveugle, je me tiens

Sur les murs aux sens effacÃ $($ ) , des dessins se chevauchent traces ind $\cong$ CChiffrables dont se jouent les rythmes du temps

nul nÃ $\bigodot$ jamais ne s'en d $\tilde{A} \odot$ tache

mais qui donc les d $\tilde{A} @$ sapprend?

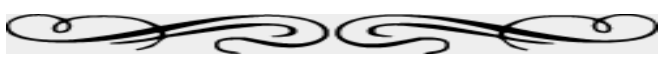

\section{Dires et Dits}

Des $\tilde{A}(c)$ critures bruissent en moi, qui voudraient naÃßtre

dont je ne sais le premier mot aux lettres entremÃa|Ã@es.

Des devenirs sourdent aux cendres projetÃ@s

lueurs parfois, gravures $\tilde{A}(\mathrm{ph} \tilde{A}(\mathrm{~m} \tilde{\mathrm{A}}$ "res graciles dans le sable de ton nom.

en retrait des ombres projettent nos d $\tilde{A} \bigodot$ sirs $\tilde{A}$ l'immuable fragilit $\cong$

des grains d'eau dorÃ@s gouttes au sable bleu o $\tilde{A}^{1}$ ne gÃ $囚 t$ aucun devenir

$\tilde{A}(g r \tilde{A}$ "nent nos avenirs

tressaillent, puis s'Ã@vanouissent ;

des vides revãatus du grÃ "s noir de nos morts assemblent leurs $\tilde{A} @$ nigmes :

" le ciel est en moi "

dit Rhader l'incroyant

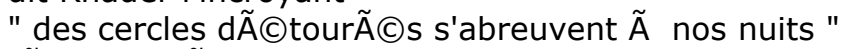
rÃ $\subseteq$ pond $H \tilde{A} @$ catis " le silence des orgues minorent nos suppliques " murmure Gabriel

mais toi de terre tu te tais

et tes pas $\tilde{A}$ l'amble se balancent

Jailli de la nuit, ton corps $\tilde{A}$ foison relie nos horizons

aux ascendants trompeurs

enceintes de nos sons des cordes vibrent que le vide habite nos gestes sont perdus 
indocile pourtant le battement de tes veines s'affaire $\tilde{A}$ vivre. 